\title{
Construyendo dominios de encuentro para problematizar acerca de las prácticas pedagógicas de profesores secundarios de Ciencias: Incorporando el modelo de Investigación-Acción como plan de formación continua*
}

\author{
Building encounter domains to problematize secondary science teachers' practice: Incor- \\ porating the action-research model as a continuous development plan \\ Construção dos domínios de encontro para problematizar ao respeito das práticas \\ pedagógicas dos professores de ciências no ensino médio: Incorporação do modelo de \\ investigação-ação como um plano de formação continuada
}

\section{Corina González-Weil, Mónica Cortez Muñoz, José Luis Pérez Flores, Paulina Bravo González y Yasnina Ibaceta Guerra ${ }^{\mathrm{a}}$}

Pontificia Universidad Católica de Valparaíso, Avenida Universidad 330, Curauma, Valparaíso, Chile. Telf.: 032- 2274859 / 032- 2274877. Correo electrónico: corina.gonzalez@ucv.cl

\begin{abstract}
RESUMEN
Frente a la actual crisis de la educación científica, la capacidad docente de transformar sus prácticas para mejorar el logro de aprendizajes parece ser fundamental. Los programas de formación docente continua tradicionales, generalmente prescriptivos y descontextualizados del aula, han tenido bajo impacto en los procesos de transformación de las prácticas docentes. El presente trabajo describe la primera fase de un programa de formación continua consistente en la construcción de dominios de encuentro entre el saber académico generado en la Universidad y el saber profesional de 16 profesores secundarios de Ciencias a través de un proceso de investigación-acción conjunta, basado en el trabajo colaborativo, la reflexión y la retroalimentación. Los resultados dan cuenta de la complejidad de generar significados comunes, los que una vez logrados, permiten fortalecer el razonamiento científico, la autoeficacia y conformación de una comunidad de aprendizaje que posibilita una actitud indagatoria hacia sus prácticas en Ciencias.
\end{abstract}

Palabras clave: educación científica, formación docente, investigación-acción.

\begin{abstract}
Considering the current scientific education crisis, teachers' capacity to transform their practice in order to improve learning achievement seems fundamental. The traditional programs of continuous professional development, generally prescriptive and uncontextualized from the classroom, have had a low impact on processes of teachers' practice transformation. This work describes the first stage of continuous development program, consisting on building encounter domains between the academic knowledge of Universities, and professional knowledge of 16 secondary science teachers, through a joint action-research process, based on collaborative work, reflection and feedback. The results show the complexity of building shared sense which, once achieved, allows strong scientific reasoning, self-efficacy and the construction of a learning community facilitating an inquiry-driven attitude towards their science teaching practice.
\end{abstract}

Key words: science education, professional development, action-research.

\section{RESUMO}

À frente de à atual crise da educação cientifica, a capacidade do docente para transformar as suas práticas para melhorar/incrementar o aprendizagem parece ser fundamental. Os programas tradicionais de formação docente continuada,

* $\quad$ El presente trabajo se desarrolla en el marco del proyecto FONDECYT N ${ }^{\circ} 11080267$. 
os quais são em geral prescritivos e descontextualizados da aula, tem tido pouco impacto nos processos de transformação das práticas docentes. O presente trabalho descreve a primeira fase de um programa de formação continuada o qual consiste na construção de domínios de encontro entre o conhecimento acadêmico gerado na Faculdade e o conhecimento profissional de 16 professores de ciências do ensino médio mediante um processo de investigação ação em conjunto, baseado no trabalho colaborativo, na reflexão e na retroalimentação. Os resultados mostram a complexidade de gerar significados comuns, os que no momento de ser alcançados permitem fortalecer tanto o razoamento científico, a autoeficácia como a conformação de uma comunidade de aprendizagem que permite uma atitude indagatória ao respeito das suas praticas em ciências.

Palavras chave: educação cientifica, desenvolvimento profissional, investigação ação.

\section{INTRODUCCIÓN}

En una cultura del aprendizaje donde predomina la construcción de conocimientos, abunda la información y se presentan condiciones de elevada incertidumbre (Pozo, 1996; Moliner y Loren, 2010), la profesión docente presenta una serie de desafíos, entre los cuales se puede destacar el que el profesor sea capaz de gestionar su conocimiento profesional de manera de transformar sus prácticas y con ello lograr mejores aprendizajes en sus estudiantes. Si se centra el análisis en la educación en Ciencias, la situación se complejiza aún más. En la actualidad, el objetivo de la educación científica es desarrollar ciudadanos "científicamente competentes", es decir, ciudadanos capaces de utilizar conocimientos y capacidades científicas para la resolución de problemas y la toma de decisiones argumentadas en ámbitos sociocientíficos (Holbrook \& Rannikmae, 2009). Adicionalmente, existe poco interés por escoger carreras de Ciencias, lo que impacta negativamente sobre el número de profesionales que se necesitan (que ya es escaso) para desarrollar el futuro tecnológico requerido para los años venideros (Lavonen \& Laaksonen, 2009; Tytler, 2007). Lo anterior podría estar relacionado con la manera en que generalmente se enseña Ciencias en la educación secundaria, una manera transmisiva y enciclopédica, poco contextualizada a la realidad del alumno y con escasas oportunidades de aproximarse de manera directa al conocimiento a través de procesos científicos (Pasmanik \& Cerón, 2005, Albertini et al., 2005). Un enfoque de enseñanza que contrasta con el formato tradicional es el de la Indagación Científica, entendida como "una enseñanza centrada en el alumno, en donde el docente orienta la construcción de conocimientos científicos en el alumnado a través de actividades concretas que involucran el poner en juego una serie de competencias relacionadas con el quehacer científico" (González-Weil et al., 2012). A partir de lo anterior, uno de los principales desafíos para los programas de desarrollo profesional docente y en particular para el caso de los docentes de Ciencias, es cómo generar la transformación de las prácticas de enseñanza. Más específicamente, ¿Cómo elaborar un proceso de formación continua con docentes secundarios de Ciencias, que logre transitar hacia nuevas formas de enseñar que permitan desarrollar altos niveles de alfabetización científica en los estudiantes?

1.1. MODELOS DE FORMACIÓN CONTINUA DOCENTE: ¿QUÉ TIPO DE FORMACIÓN CONTINUA DOCENTE SE REQUIERE PARA TRANSFORMAR PRÁCTICAS PEDAGÓGICAS EN EL ÁMBITO DE LA ENSEÑANZA DE LAS CIENCIAS?

Copello y Sanmartí (2001) plantean que los modelos de formación continua se sitúan en dos grandes tipologías. La más extendida consiste en la realización de cursos sobre temas específicos o nuevas orientaciones, en la línea de un perfeccionamiento, el que por 
lo general está alejado de la práctica concreta. La otra consiste en la formación en el propio centro de trabajo, a partir del planteamiento de temáticas susceptibles de interesar a todo el profesorado. Vezub (2007), por su parte, señala que la formación continua de los docentes aun es pensada desde una lógica lineal, donde primero se define el sentido de la transformación que se desea y luego se diseñan los cursos correspondientes. En cualquier caso, las acciones formadoras deben apuntar a que los docentes logren una fundamentación teórica de su actuación, congruente con los nuevos conocimientos o niveles reflexivos en relación al proceso de enseñanza-aprendizaje de las Ciencias que irán elaborando, lo que a su vez debe ir estrechamente vinculado a sus prácticas. Asimismo, es necesario que se den cambios en el sistema de valores y actitudes, facilitados por una reflexión crítica tanto en relación con la forma actual de enseñar Ciencias como a las posibles innovaciones planteadas (Copello \& Sanmartí, 2001). Una constante en las experiencias exitosas en formación continua parece ser el ofrecer espacios de trabajo colaborativo, donde se promueva la reflexión y el cuestionamiento de las propias creencias y prácticas a partir de la discusión y el intercambio de experiencias (Park Rogers et al., 2007; Vezub, 2007). Un ejemplo concreto es el estudio de Furió y Carnicer (2002), quienes a partir de este tipo de espacio formativo lograron un cambio epistemológico, respecto a la Ciencia y su enseñanza, un cambio metodológico en su comportamiento docente y un cambio actitudinal hacia la investigación didáctica y en las expectativas de desarrollo profesional.

De esta manera se transforma la visión de la profesión, desde un trabajo solitario en el aula hacia una visión basada en el profesionalismo colectivo que implica que los profesores sean convocados a trabajar y aprender en equipo (Montecinos, 2003). Se requiere un docente indagatorio respecto a sus prácticas y comprometido con su aprendizaje profesional continuo y colectivo (Montecinos, 2003). En el caso particular de los docentes de Ciencia, el concepto de indagación adquiere un significado más allá de su posible implementación en el aula, al ser comprendido como un enfoque indagatorio, donde los profesores se cuestionan sus propios conocimientos y su práctica y colaboran en el cuestionamiento de los conocimientos y las prácticas de otros, para transformar su práctica desde una postura crítica y transformadora (Cochran-Smith \& Lytle, 2003). La Indagación Científica a este nivel constituye una "orientación hacia la reflexión" (Abell et al., 2006), donde el docente indaga sus propias prácticas, para luego trasladar este proceso reflexivo y de indagación a la construcción de conocimiento científico por parte de sus alumnos.

\subsection{LA INVESTIGACIÓN ACCIÓN Y SU APORTE A LA FORMACIÓN CONTINUA DE DOCENTES}

Considerando los aspectos mencionados anteriormente, se plantea a la InvestigaciónAcción como una metodología pertinente para abordar un proceso de formación, a la vez que presenta sinergia con el concepto de Indagación Científica como enfoque sugerido para la enseñanza de la Ciencias. La Investigación-Acción promueve en los profesores la búsqueda de nuevas comprensiones y conocimientos de su práctica pedagógica, al mismo tiempo que les permite resolver problemas que les impiden optimizar el aprendizaje de sus alumnos (Montecinos, Solís \& Gabriele, 2001), convirtiéndose en un espacio para la reflexión, el diálogo entre pares y la construcción conjunta de conocimientos (Labra et al., 2005). 
En este contexto la apuesta es conformar una comunidad de profesores aprendices que construyan colectivamente la comprensión de sus quehaceres y estrategias profesionales, mediante la reflexión deliberativa colaborativa (Elliot, 2010). Donde los guías de la capacitación tengan más un perfil práctico reflexivo, que ayude a que sean los propios docentes quienes diagnostiquen sus dificultades y avancen hacia posibles soluciones, más que ser acompañantes expertos infalibles que solucionen los problemas de otros (Imbernón, 2002 en Moliner \& Loren, 2010). El presente estudio explora el desarrollo de la primera fase de un proceso de Investigación-Acción, realizado por el equipo de investigación del Laboratorio de Didáctica de la Biología de la PUCV y 16 docentes secundarios de Ciencias, quienes se unieron para mejorar sus prácticas de enseñanza, a través de la problematización y transformación de éstas. A partir de este trabajo, se espera contribuir a la comprensión de los procesos de desarrollo profesional continuo y, a la vez, dar luces para la elaboración de este tipo de programas de formación docente.

\section{METODOLOGÍA}

El presente estudio adhiere a una metodología de Investigación-Acción y se centra en la primera fase del proceso: la reflexión inicial. Para Elliot (2010) el propósito fundamental de esta primera fase consiste en que el profesor pueda profundizar la comprensión de su problemática, adoptando una postura de exploración y cuestionamiento de las explicaciones iniciales que se da respecto al problema. Para lograr este propósito se requiere la creación de espacios sistemáticos para la reflexión, revisión y elaboración de la teoría emergente, siendo necesario generar las condiciones psicológicas y de organización adecuadas, así como contar con procedimientos básicos de investigación. Esta primera fase, que en este caso duró 8 meses, es indispensable para la posterior implementación de acciones en aula, considerando que la comprensión profunda de dicha problemática es el eje fundamental para una óptima intervención. El análisis que se presenta es resultado de una Investigación-Acción de segundo orden, es decir, una "investigación en la investigación", realizada por el equipo en su conjunto y que pretende dar orientaciones para la elaboración de un Plan de Formación Continua de docentes secundarios de Ciencias a través de esta metodología.

\subsection{BREVE DESCRIPCIÓN DEL PROYECTO}

El proceso presentado se enmarca dentro de un proyecto de investigación que tiene como objetivo principal desarrollar y evaluar, en el marco de una Investigación-Acción, un plan de formación continua basado en la Indagación Científica como enfoque pedagógico (ver González-Weil et al., 2012), para profesores secundarios de Ciencias (Biología, Química y Física) pertenecientes a establecimientos Municipales y Subvencionados de la Región de Valparaíso, que apunte al desarrollo de Competencias Científicas en sus estudiantes y sea pertinente al contexto y necesidades de aprendizaje de los profesores involucrados. El proyecto se inició en Junio de 2010 e implicó sesiones semanales de 3 horas de trabajo. Las sesiones abordaron temas como: desafíos actuales de la enseñanza de las Ciencias, imagen de enseñanza efectiva de las Ciencias, investigación acción en educación, desarrollo de Competencia Científica e Indagación Científica como enfoque de enseñanza. El foco de la primera etapa fue la construcción de significados comunes 
para trabajar. Posteriormente, las sesiones abordaron el proceso de Investigación Acción realizado por cada profesor en su aula. El análisis de los resultados de las fases de acción e implementación en el aula, así como la evaluación final del proceso se encontraban en curso al cierre de este reporte.

\subsection{PARTICIPANTES}

El grupo de trabajo estuvo conformado por 16 docentes de Ciencia secundarios, de Química (6), Biología (5) y Física (4), además de un profesor de Ciencia aplicada, ${ }^{1}$ provenientes de 7 comunas de la Región de Valparaíso. Todos poseen experiencia laboral que va desde 5 a 40 años de docencia. Entre el grupo, hay representantes de establecimientos educacionales de distinta dependencia: Estatales (1), Municipales (9), Subvencionados (5), Particulares (1). Junto a ellos, trabajó el equipo de investigación de la Universidad, compuesto por una didacta de las Ciencias, una psicóloga y una bióloga, a las que se sumaron, en algunas sesiones, una profesora de biología y otra psicóloga.

\subsection{FUENTE DE DATOS}

Los datos para este estudio fueron obtenidos a partir de las filmaciones, transcripciones, registros y materiales de cada una de las 21 sesiones (aprox. 63 horas), focus group realizados con los profesores y productos escritos de los docentes (informes, bitácoras, tareas). Estos materiales fueron analizados y codificados a nivel descriptivo y analítico.

\section{RESULTADOS}

Los resultados de este trabajo se organizan en tres niveles: (a) Descripción del proceso y el respectivo análisis de las etapas (b) Análisis de segundo orden, relativo a evidenciar ámbitos de impacto en los docentes y factores que contribuyeron a generar dicho impacto y (c) Síntesis de los resultados a partir de la articulación de los dos niveles anteriores.

\subsection{NIVEL I. DESCRIPCIÓN DEL PROCESO}

\subsubsection{Fase O: Generando dominios comunes para problematizar}

Por el carácter de este proceso de investigación, una primera fase se centró en poder establecer una alianza de trabajo entre el equipo de profesionales del proyecto $\mathrm{y}$ los profesores invitados a participar. Esta primera parte implicó el desafío de constituir un equipo de Investigación-Acción y compartir un proyecto común. Este momento del proyecto quedó reflejado en lo que ha sido denominado como "Escena 1".

Dicho docente trabaja en un Liceo Técnico Estatal, enseñando fundamentalmente mecánica automotriz, aplicando básicamente conocimientos de física y química. Si bien no estaba dentro de las expectativas del proyecto, se incorporó debido a su experiencia de aplicación de conceptos científicos a la vida práctica. 


\subsubsection{Escena 1: La realidad es otra: "lo hemos intentado todo y la brecha crece"}

El primer (des) encuentro entre el equipo de profesionales y los profesores, refleja con claridad esta primera escena. Dicho encuentro se realiza en un auditórium de la Universidad donde se expone en qué consiste el proyecto a través de una presentación y una breve actividad. Las opiniones de los docentes reflejan desesperanza, cuestionamientos, resistencias y condiciones. A pesar de este panorama, se aprecia compromiso con el tema:

“...Como lo acaban de señalar las colegas (apuntando hacia las otras participantes) la realidad es otra, el hecho de tener cero resultado con los alumnos, que los alumnos no quieran nada de nada, es muy frustrante y preocupante; uno ve para donde va la sociedad o parte de ella. Esta brecha que hay entre colegios privados y municipalizados, crece a pasos agigantados y los profesores, es la discusión diaria; ¿qué podemos hacer? Muchas veces no sabemos qué hacer, lo hemos intentado todo y la brecha crece y nosotros ni siquiera podemos mantener una situación paralela, a pesar de eso, todavía me quedan ganas para seguir luchando" (S1; M5). ${ }^{2}$

"Dos acotaciones que me preocupan: una tiene que ver con este proceso de investigación en el cual los docentes puedan colaborar, yo creo que hay que focalizar las cosas, que des una claridad, o trates de aclarar, qué pasa con la cantidad de horas, como dice el colega, a muchos docentes, y me incluyo, en la práctica no nos sirve de nada una investigación si efectivamente no es reconocida por nuestros sostenedores. Esa cuestión es clave. Una segunda cosa que yo creo ganaría bastante plus entre los docentes es el reconocimiento de la labor que se hizo, porque si tú solamente lo asocias, y no lo digo sólo por esta situación, si sólo lo asocias a una suerte de investigación, nadie lo va a hacer"(S1;M4 ).

“...Una consulta ¿qué tanta experiencia tienen ustedes? El equipo junto a la psicóloga, de trabajar en un Liceo, trabajar, no visitar, o sea, te pongo la experiencia de los profesores. En el tiempo que estuve en España, todos trabajan en liceos como los nuestros, tenían una experiencia real, no teórica, porque realmente, después de tanta experiencia y tanto curso, estoy absolutamente convencido que el único especialista en didáctica de la Ciencia, o en la que sea, es el profesor del aula, nadie más, todos los que están afuera pueden tener muchas teorías, algunas de las cuales son muy buenas, y que uno debe, necesariamente, conocer para ver si las puede aplicar, pero el único que dice sí esto lo puedo hacer, o no lo puedo hacer, es el profesor del aula. Sería muy bueno que ustedes dijeran, ya, voy tres años al liceo a trabajar, voy a trabajar con un profesor común y corriente ahí. $Y$ después vengo con todas estas cositas para acá...” (S1; M5).

Como se desprende de las citas, los profesores intentan traer "su realidad, su cotidianeidad" a escena, describen con insistencia la realidad que viven y entregan sus experiencias de investigación anteriores. De esta forma sitúan en la escena la experticia con la que cuentan y que asumen el equipo de investigación no posee. Esta situación genera en el equipo de investigación una serie de reflexiones respecto al setting utilizado: espacio físico (la universidad), lugar (auditórium), presentación (en PowerPoint), entre otras. Cada uno de estos elementos configuraron un escenario que dio señas a los profesores de que éste sería un proceso de capacitación (y seguramente con ello, el recuerdo de varios cursos a los que han asistido), con características totalmente opuestas a la idea del proyecto de un trabajo de Investigación-Acción Colaborativa. Otro elemento presente en la discusión

2 S1 corresponde a la Sesión 1 y M5 (Mx) corresponde al código del profesor participante. 
eran las dudas que quedaban respecto a la comprensión del proyecto presentado ¿Se logró compartir el propósito y los sentidos de la investigación? Esta inquietud se origina debido a que en las intervenciones de los profesores no aparecieron preguntas respecto al proyecto y solo se abordaron aspectos tangenciales o administrativos.

Frente al desánimo de este primer acercamiento, se volvió imprescindible re-pensar cómo generar un mejor espacio de trabajo. Un elemento a favor era que a pesar del diagnóstico pesimista y de las resistencias presentadas, los docentes finalizaban sus relatos con frases que aludían disposición de ser partícipes del proyecto, aspecto que queda reafirmado cuando la totalidad de los participantes asisten a la segunda sesión. Se decide entonces introducir varios cambios a la forma de realizar las reuniones, de manera de propiciar un espacio de trabajo que invitara a una relación horizontal y dialógica. Se opta, entonces, por la utilización de una mesa de trabajo circular que permitiera el trabajo en equipo, suspender el uso de presentaciones formato PowerPoint, hacer lectura del proyecto individual y luego compartir en pequeños grupos, para finalizar en un plenario general. El cambio generado es evidente; se aprecia una elevada participación de los profesores, esta vez centrada en el proyecto y no solo en aspectos administrativos.

En las sesiones siguientes se siguió propiciando un ambiente de trabajo que posibilitara la constitución de un equipo de Investigación-Acción. El nuevo desafío que había que afrontar era comenzar a generar un lenguaje compartido que permitiera dialogar y construir un proyecto común. En este momento surge una nueva escena.

\subsubsection{Escena 2: ¿De qué hablamos cuando hablamos de Indagación, Competencia Científica e Investigación-acción?}

Esta escena refleja la dificultad de establecer un diálogo cuando los participantes presentan diferencias en la comprensión de los conceptos centrales del proyecto. Hay confusión respecto al concepto de Indagación Científica. ¿Qué se entiende por Indagación? ¿Cuál es su relación con el constructivismo? ¿Cuál es la relación entre Indagación y contenido disciplinar?, entre otras.

Los docentes presentan elevado desconocimiento en ámbitos de actualización a nivel de concepto de Indagación Científica, donde se evidencia bastante confusión; y de Competencia Científica que, de acuerdo a los análisis realizados, parece ser un tema bastante ambiguo para los profesores.

En torno a la Indagación Científica: Los profesores, en general, desconocen el concepto o lo comprenden como una atribución propia del alumno (capacidad). A menudo se remiten al proceso de búsqueda de información, particularmente búsqueda bibliográfica, al modo en que se expresa en los libros de texto, o bien a un proceso orientado al desarrollo de capacidades, pero no de conceptos, lo que produce tensión de tener que optar entre uno u otro.

"Cuando se nos preguntó sobre la comprensión del concepto, no dudé en pensar que indagación científica se refería a un tipo de investigación bibliográfica que debía ser ejecutada por los alumnos a partir de un problema planteado por el profesor" (CON; M8). ${ }^{3}$

CON: Consulta escrita a docentes 
Estudios Pedagógicos XXXIX, No 2: 129-146, 2013

CONSTRUYENDO DOMINIOS DE ENCUENTRO PARA PROBLEMATIZAR ACERCA DE LAS PRÁCTICAS PEDAGÓGICAS DE PROFESORES SECUNDARIOS DE CIENCIAS...

“...Estábamos ahí viendo que si nosotros queremos hacer solamente indagación, requiere de bastante tiempo, y nosotros vamos para otro lado, con un programa de estudio, entonces, ¿cómo lograrlo? ¿en qué lugar lo colocábamos? Por eso estábamos con la duda, porque el profesor tiene que ser flexible en su aula, tiene que ser capaz de ver cuándo inserta esta clase frontal, que de hecho no es negativa, y las otras técnicas del constructivismo para poder congeniar ambos aspectos y para que la clase sea efectiva también tiene que tener conceptos netos" (S6; P2).

En torno a la Competencia Científica: En un comienzo, parece un concepto difuso, asociado por una parte a una característica propia de cada individuo (p. ej., curiosidad), o vinculado principalmente a una "actitud" o "capacidad científica". En el momento de hacer el ejercicio grupal de pensar qué elementos componen la Competencia Científica, la mayoría de los profesores se centra en aspectos actitudinales como: disposición al aprendizaje, valorización del conocimiento científico, autonomía, entre otros; seguida por competencias del saber hacer: rigurosidad científica, habilidades de expresión oral y escrita, procedimientos de experimentación, revisión bibliográfica y habilidades metacognitivas, entre otras. Sin embargo, se produce gran dificultad al abordar el ámbito del saber referido a los conocimientos, donde con algunos obstáculos se arriba a considerar los conocimientos previos, conocimientos matemáticos básicos, lectura comprensiva y lenguaje científico, pero sin abordar conceptos centrales. ¿Por qué sucede esto? ¿Se debe a la multidisciplinareidad del grupo?¿Se debe a un curriculum más centrado en el saber hacer y ser o a que éste es un aspecto en el que el docente no tienen facultad de incidir, debido a que está determinado por el currículum? En contraposición a lo anterior, ¿a qué se deberá que aparezca rápida y masivamente el ámbito actitudinal? ¿Refleja éste la principal preocupación de los docentes?

En la siguiente sesión se aborda nuevamente el tema solicitándoles que refieran los tres conceptos nucleares que debe aportar su disciplina en los años de educación secundaria. Los profesores discuten respecto a cómo se entrelazan los contenidos de las tres disciplinas y el escaso trabajo conjunto que se realiza entre ellas en las escuelas, reconociendo que existen ciertos conceptos que son transversales, como energía, transferencia de energía, entre otros

En torno a la Investigación Acción: Aquí, la mayoría de las inquietudes se centraban en escepticismo respecto a su aporte, a la capacidad de los docentes de inspeccionar sus prácticas y a la ausencia de herramientas para poder desarrollar un proceso de investigación:

"Se hace la pregunta, ¿qué significa que investigue mis prácticas? ¿Qué herramientas básicas manejo para desarrollar una investigación? Y ahí no avanzamos, porque no tengo las estrategias metodológicas, nadie me las enseñó, porque no se enseña en ninguna carrera de formación docente, cómo investigar, qué significa investigar las prácticas docentes, investigar mi aula, entonces ahí yo creo que se frena todo; y lo otro, luego, si es que hubiera un profesor excepcional se encuentra con todo el bloqueo temporal, ambiental de sus propios colegas que sería incomprendido en una situación de investigación; que él se preste o le pida a otro la posibilidad de investigar es bastante complejo"(S8; P1).

\subsubsection{Escena 3: Construyendo dominios de significados comunes}

a través de la discusión grupal "¿Estará bien decir que no estamos hablando de una metodología? (refiriéndose a la Indagación)"

Un aprendizaje relevante que tomó varias sesiones de trabajo fue la construcción de un dominio de significado común en torno a estos conceptos claves para el proyecto. 
Dichos significados fueron construidos colaborativamente y permitieron seguir avanzando en el proceso.

En torno a la Indagación Científica: De lo individual, a lo grupal y a lo colectivo. A través de un trabajo exhaustivo con los profesores participantes, se logra avanzar de definiciones individuales a grupales y, por último, a una definición colectiva. En las definiciones grupales realizadas por los profesores se encontraron elementos comunes que posibilitaron esta construcción final. Una de las dimensiones referidas fue la presencia de un rol distinto del profesor y el alumno en la Indagación. Esta definición posiciona al docente como un facilitador del aprendizaje y, por ende, los profesores visualizaban que se requería de elevadas habilidades reflexivas, buen dominio de la disciplina científica y capacidad de adecuar de manera flexible las actividades. Dicho rol generaba cuestionamiento e inquietud entre los profesores:

"Bueno, primero lo que le ocurre al alumno es un proceso dinámico y de participación del alumno, donde el desarrollo es más activo, permite generar un alto grado de conocimiento y a la vez permite contrastar experiencias anteriores; el contrastar con situaciones anteriores te permite de nuevo volver al círculo y se hace más dinámico, eso de parte de la visión que tengo del alumno; ahora, la visión que tengo de parte del profesor es que debe ser un estratega, en su desarrollo y en su concepción, pero aquí hay un escalamiento terriblemente fuerte, lo que significa que para poder llegar a eso las habilidades del profesor tienen que ser de una manera dada, contar con elementos para que él pueda, de alguna manera jugar, eso yo veo que es complicadísimo, o sea, veo que es un tema fuerte, donde la formación de los profesores no está dada así, uno puede enfrentar situaciones de aula, como, estoy pensando en una clase $x$, donde uno tiene que improvisar ciertas situaciones, pero esto es fuerte, yo diría que la situación indagatoria involucra un trabajo del docente que es bastante complejo, estamos claros que nosotros somos profesores de Ciencia y tenemos un manejo, bueno, se supone, de lo que es la investigación científica, pero aquí hay una cosa que escapa a eso, entonces eso yo creo que es complicadísimo" (S10; M4).

Respecto al rol de alumno, para los profesores implica una participación activa, que conlleva como resultado o producto la adquisición de nuevos conocimientos y/o modificación de conocimientos previos.

"Yo creo que (la indagación) es la adquisición de nuevos conceptos a partir de conocimientos previos que le van a permitir al alumno modificar lo que ya tenía, a la concepción que ya tenía de algo y le va a permitir una nueva forma de ver el mundo" (S10; M8).

Otra dimensión compartida por las definiciones de los profesores, era la comprensión del enfoque de indagación como una metodología o estrategia que conlleva la confrontación del alumno con una situación problemática que genera un conflicto cognitivo, que puede darse o no a través de una actividad experimental.

"Coloqué lo siguiente: es una metodología que desafía lo confrontacional, donde a partir de una situación problemática se posiciona al alumno en relación a un fenómeno natural, pone a prueba sus conocimientos previos como también a situaciones de la vida cotidiana, donde todas sus ideas son válidas, donde, a través de pistas lo desafía nuevamente, en relación a corroborar lo que se predijo en una forma experimental o puede ser una forma donde el alumno trabaje con una lectura científica, donde la idea es generar nuevos conocimientos, por medio de un posible desajuste cognitivo que lleve o genere una reflexión a los nuevos conocimientos, $y$ así poder llevarlos a desafiar nuevamente una nueva problemática...” (S10; S7). 
Estudios Pedagógicos XXXIX, No 2: 129-146, 2013

CONSTRUYENDO DOMINIOS DE ENCUENTRO PARA PROBLEMATIZAR ACERCA DE LAS PRÁCTICAS PEDAGÓGICAS DE PROFESORES SECUNDARIOS DE CIENCIAS...

A partir de las discusiones generadas en la sesión, se vislumbró en los profesores el inicio de un proceso de cuestionamientos e intuiciones comunes respecto a su comprensión del concepto, el que además, comenzó a esbozarse como una posibilidad de desarrollo profesional, al momento de asumir el profesor el rol de "indagador" de sus propias prácticas:

"Yo lo vi acá, lo subrayé, lo veo ahí y también veo que aparece muchas veces y también lo comentaron recién los profesores, yo estoy en la duda, o sea, no sé si estoy en la duda, estará bien plantear decir que no estamos hablando de un proceso, estará bien decir que no estamos hablando de una metodología, ¿me entiendes? ¿o no? Porque evidentemente sumamos las diferentes visiones que podemos tener, en esta situación indagatoria no estamos hablando de pasos..." (S10; M4).

"Claro, pero, por eso mismo, ese mismo método o la forma en lo cual lo estamos aplicando en nuestro mismo proceso de crecimiento profesional, así como también lo puedes aplicar en la sala con una pregunta: ¿Qué diferencia que el alumno pueda ir avanzando con esa metodología y tú con esta metodología? Por tanto, no sé si utilizar la palabra metodología, porque a lo mejor no es el caso tal como tú dices..." (S10; M4).

Este proceso confluye en la construcción común de una definición de Indagación Científica compartida por el grupo de docentes. Ésta se entiende desde el colectivo como "un enfoque crítico a un problema que conlleva a una reflexión continua a partir de evidencias que tiene como consecuencia el desarrollo de competencias (científicas/ docentes)".

Esta comprensión colectiva final quedó expresada plenamente en el desarrollo de una Jornada de Reflexión, en donde se dio a conocer el concepto de Indagación Científica como un concepto transversal que posibilita la construcción de conocimiento, tanto en los alumnos (conocimiento científico) como en los docentes (conocimiento pedagógicodidáctico), que involucra una actitud permanente de problematización, búsqueda de evidencias y elaboración de respuestas a través de un trabajo colaborativo de construcción de conocimiento con otros.

A través del trabajo con los docentes, se fue profundizando la comprensión del concepto de Indagación Científica que tenía el equipo de investigación, el que pasó de entenderse como dos procesos separados (a nivel de enseñanza y a nivel de desarrollo profesional) con algunos elementos comunes, a un concepto transversal, de aproximación y construcción colaborativa de conocimientos a partir de una situación problemática.

En torno a Competencia Científica: Si bien no se llegó a una definición colectiva, sí hubo acuerdo en que el concepto de Competencia Científica implica la consideración de conocimientos, capacidades y actitudes, que de modo integrado se movilizan en la toma de decisiones de cuestiones relacionadas con la Ciencia.

“...Yo como profe siempre veía mis objetivos y eran conocer, comprender, aplicar...cuando miré y leí sobre competencia me di cuenta que lo que le estoy enseñando a mis alumnos le tiene que servir. Conocer leyes de Newton para mí es espectacular, pero ¿para él?...entonces el valorar, no es, necesariamente, el que uno entiende como el respeto, actitudes positivas... sino es reconocer la importancia de ese conocimiento para su cotidianeidad...me puse a pensar en el alumno... ¿cómo desarrollo el currículum de primero medio?.... y ahí me dí cuenta que tenía que pensar en otras cosas..." (S14; S5).

Del mismo modo, se discutió acerca de lo complejo que resulta evidenciar el desarrollo de Competencia Científica en los alumnos, reconociendo poca preparación para desarrollar instrumentos que puedan evaluar este aspecto. Respecto al concepto de 
Investigación-Acción Colaborativa, se pueden apreciar avances en la fase de problematización e identificación de las problemáticas que se presentan a continuación.

\subsubsection{Fase Primera: Explorando y problematizando nuestras prácticas}

Una vez que se ha establecido un dominio de significados compartido y una carta de navegación, que contempla un propósito común a través del cual avanzar en esta investigación, empieza el desafío de comenzar a explorar un plan de formación profesional basado en la metodología de Investigación-Acción, que incorpore el enfoque de indagación como postura para promover las competencias científicas de los estudiantes. La fase previa, que contempló aproximadamente 5 meses de trabajo, refleja la distancia que existe en ocasiones entre la producción académica del mundo universitario y el conocimiento adquirido en el ejercicio profesional por los docentes de educación secundaria.

El proyecto de Investigación-Acción comenzó con el acuerdo de que cada profesor identificara y desarrollara una descripción de un problema de aula que limitara su ejercicio profesional en la enseñanza de las Ciencias en educación secundaria. Para dicho fin, cada profesor realizó un diagnóstico exhaustivo de la problemática escogida, en base a un análisis crítico de la evidencia recopilada y a la incorporación de elementos teóricos actualizados acerca del problema. Los análisis, basados en 14 informes de los 16 docentes participantes, muestran que la mayoría de dichas investigaciones $(64 \% \mathrm{~N}=9)$, planteó como principal dificultad la disposición y el compromiso deficiente de parte de los estudiantes al enfrentar el proceso de aprendizaje. Más de la mitad de estos estudios (56\% N=5) refiere que esta actitud de los estudiantes se expresa particularmente hacia el aprendizaje de las Ciencias, de este modo, este último grupo de estudios abordaría propiamente el ámbito actitudinal de la Competencia Científica definido por PISA (OCDE, 2006).

"La problemática identificada es la falta de una actitud responsable en el estudio de las Ciencias, ya que el fomentar en los alumnos una postura o actitud responsable les ayudará a lograr metas, estabilidad en sus relaciones y objetivos en cualquier ámbito de su vida, ya sea en su autocuidado, así como también, en la responsabilidad con el medio". (RE; M8). ${ }^{4}$

De las problemáticas que abordaban el ámbito actitudinal, un $67 \%(\mathrm{~N}=6)$ pertenecían a docentes de establecimientos municipalizados, situación que implica que el interés de estos docentes se centre más bien en aspectos "actitudinales transversales", en desmedro de los otros dos ámbitos de la Competencia Científica. Del resto de las investigaciones, sólo un $21 \%(\mathrm{~N}=3)$ centra su problemática en las incipientes capacidades que presentan los estudiantes para un buen proceso de construcción de aprendizaje en Ciencias, refiriendo como problemas: insuficiente desarrollo de pensamiento crítico, autonomía y creatividad, dificultades para aplicar los conocimientos adquiridos en evaluaciones y débiles habilidades de comprensión lectora en ciencias.

"El ítem de desarrollo en las evaluaciones escritas es el de más bajo rendimiento, donde los alumnos deben demostrar creativamente soluciones a problemas planteados......Algunos trabajos son fieles copias de compañeros o de Internet. El alumno, de alguna manera, presenta inseguridad en la toma de decisiones de sus planteamientos, pues no está acostumbrado a la consulta bibliográfica, extracción de una buena fuente de información, copian mucho de la pizarra, no están acostumbrados a tomar apuntes" (RE; S2).

4 RE: Reporte escrito; código docente. 
Estudios Pedagógicos XXXIX, No 2: 129-146, 2013

CONSTRUYENDO DOMINIOS DE ENCUENTRO PARA PROBLEMATIZAR ACERCA DE LAS PRÁCTICAS PEDAGÓGICAS DE PROFESORES SECUNDARIOS DE CIENCIAS..

El 14\% de los profesores ( $\mathrm{N}=2)$ aborda el insuficiente conocimiento o dominio conceptual en el ámbito de las Ciencias que presentan los estudiantes como base para generar nuevos aprendizajes, lo que genera un abismo entre el dominio conceptual (significados) que posee el profesor y el dominio temático del estudiante.

"Estos dos antecedentes me señalan que la problemática está presente y que debo preocuparme y reflexionar ¿por qué es tan baja esta adquisición del conocimiento? Subir porcentualmente este nivel de logro trae aparejado varias aristas positivas, partiendo de una simple, como que el alumno llegue a comprender de qué cosa le están hablando en el ramo de Física, hasta que pueda ser capaz de comprender a no tan largo plazo y lograr de explicar con sentido crítico diferentes fenómenos que ocurren en su entorno y si sigue aumentando sus capacidades científicas, puede llegar a vivir con responsabilidad como ciudadano" (RE; M4).

La mayoría de los profesores, un $71 \%(\mathrm{~N}=10)$, recolectó evidencia a partir de documentos, recopilando indicadores educativos nacionales y/o datos consignados en registros propios de los Establecimientos. Un 29\% ( $\mathrm{N}=4)$ de las investigaciones recopilaron información a partir de reportes de actores educativos, destacando la utilización de encuestas y entrevistas diseñados por los propios docentes. Un 21\% ( N=3) incorporó en su recolección de información la aplicación de pruebas estandarizadas y sólo un 7\% $(\mathrm{N}=1)$ realizó observación de sus clases.

A partir de estos datos se aprecia que la mitad de los profesores analiza la problemática contemplando una sola fuente de información, es decir, fundamenta su problemática desde el análisis de un documento o en base a la opinión de un solo actor educativo. De quienes contemplan opiniones de actores educativos, encontramos que un $36 \%(\mathrm{~N}=5)$ contempla las creencias de sus colegas y un número menor de $21 \%(\mathrm{~N}=3)$, incluye a los estudiantes al momento de indagar acerca de la problemática identificada. Esta información dio cuenta de la necesidad de potenciar aún más el acercamiento de los docentes hacia otras fuentes de información, particularmente a actores educativos claves como son los estudiantes, docentes, directivos, apoderados, entre otros. Para esto fue necesario problematizar el tema con los profesores, fortalecer las estrategias metodológicas de recopilación de información y robustecer la calidad de los análisis. La realización de presentaciones orales donde cada profesor exponía su problemática deteniéndose en la evidencia que la sustentaba, posibilitó la discusión de los profesores respecto a la relevancia de incorporar la "voz" de otros actores, especialmente la de los estudiantes, por cuanto la mayoría de las investigaciones se centraba en problemas en el ámbito actitudinal. También se señala el interés por incorporar a un observador externo que fuese a registrar a las aulas. Se debe destacar que, a pesar de que los docentes quisieran incluir otras fuentes de información para sustentar su problemática, el tiempo disponible para abordar este aspecto fue escaso, por lo que resulta relevante señalar que las acciones que se pudieron realizar respecto a las fuentes de información son el resultado de un trabajo constante y de mucho compromiso, por parte de los docentes, con el proceso.

Las destrezas de los profesores para auto-inspeccionar sus prácticas fue un ámbito clave para el desarrollo de esta primera etapa, sin embargo, implicó un trabajo arduo, debido a que las hipótesis o explicaciones sostenidas por los profesores respecto a las problemáticas identificadas inhibían la exploración de sus propias prácticas al realizar atribuciones que tendían rápidamente a ubicar el problema en factores externos macro estructurales (sistema educativo, tiempo para planificación) o en factores externos a nivel micro (los estudiantes). De ahí que el trabajo consistió en fortalecer las destrezas de auto-inspección 
de los profesores, a partir de discusiones grupales, ejercicios, retroalimentaciones de los trabajos escritos y asesoría a cada una de las investigaciones. Por medio de esos trabajos se lograron interesantes avances que quedan reflejados en sus bitácoras de trabajo, en los informes escritos, en las presentaciones orales de sus trabajos al interior del equipo y en seminarios abiertos a profesores de Ciencias en educación secundaria. Se aprecia una profundización en los niveles de análisis y una mejor calidad de la argumentación a partir del proceso de investigación. De ahí que un logro interesante de esta etapa sea que 14 de los 16 profesores elaborara su informe final escrito (auto reporte) con reflexiones y discusiones a partir de la evidencia recopilada.

\subsection{NIVEL II: ANÁLISIS DEL IMPACTO EN TRES ÁMBITOS DEL DESARROLLO PROFESIONAL}

Hasta el momento se distinguen, a lo menos, tres ámbitos donde se aprecia que este proceso de investigación ha generado impacto:

\subsubsection{Potenciamiento del razonamiento científico de los docentes}

En un comienzo los profesores, al argumentar sus planteamientos, ignoraban otros factores presentes en su problemática; incluso, frente a los primeros datos obtenidos, los distorsionaban con el propósito de hacerlos coincidir con sus hipótesis o explicaciones previas. El argumento que predominaba en los profesores al iniciar la investigación se sintetiza en la idea "Los estudiantes no están motivados a aprender Ciencia", lo que los lleva a focalizar su problemática en un "otro" (los estudiantes), donde no tienen posibilidad de incidir, quedando inmovilizada su acción profesional. Durante el proceso de diagnóstico de la IA (recopilación y análisis de evidencias) así como las discusiones grupales, se logra transitar hacia otra explicación: "no hemos sabido motivar a los alumnos para que se interesen en las clases de Ciencia", argumento que incorpora en el análisis al actor profesor como agente capaz de desplegar acciones que posibiliten incidir en el interés de los estudiantes en la clase de Ciencia. Un grupo de docentes $(64 \% \mathrm{~N}=9)$ va más allá y deja entrever en su informe final un argumento explicativo centrado en dilucidar junto a sus estudiantes la relevancia de aprender Ciencias. Si el profesor reflexiona acerca del para qué, entonces es capaz de argumentar y construir junto a sus estudiantes el sentido del aprendizaje de las Ciencias y desde ahí redireccionar el cómo debe hacerlo para asegurar que esto se logre considerando las condiciones contextuales en las cuales enseña. Al posicionarse desde este argumento, los profesores comenzaron a sentir la necesidad de analizar la problemática, contemplando las distintas perspectivas que confluyen en el problema (estudiantes, profesores, contexto, demandas de la sociedad actual, etc.), logrando identificar en cuál de ellas pueden incidir como profesores.

\subsubsection{Fortalecimiento de la Autoeficacia}

Una de las dimensiones que incide en la transformación de las prácticas, tiene relación con abordar aquellos aspectos denominados como mediadores psicológicos (Coll et al., 2004). Uno de los mediadores fundamentales es la autoeficacia para la enseñanza debido a que si los profesores se perciben capaces de realizar de buena manera su quehacer profesional, logran mejor desempeño y mejores resultados en sus estudiantes 
(Yilmaz-Tuzun y Topcu, 2008; Knobloch, 2006 en Garduño et al., 2010). La autoeficacia es el juicio personal acerca de la propia capacidad para realizar una tarea. En las expectativas de autoeficacia inciden las experiencias de éxito previo, la experiencia vicaria, la persuasión verbal y el estado emocional (Bandura, 1987). En las opiniones referidas por los miembros del equipo, aquella que más se destaca es el haber generado un ambiente de trabajo colaborativo que justamente repercute en los ámbitos señalados por Bandura (1987); los profesores lograron compartir sus buenas experiencias en la enseñanza de las Ciencias, escuchar las buenas prácticas y buenos resultados de otros colegas, así como las experiencias de fracaso que permitieron consolidar aprendizajes. Fueron retroalimentados por sus pares, quienes reforzaron las buenas ideas y prácticas. Todo este trabajo se realizó en un ambiente en que prevalecía el respeto, la escucha activa, apertura mental y la crítica constructiva, lo que promueve estados emocionales agradables que favorecen el aprendizaje. Una actividad central que permitió el fortalecimiento de autoeficacia en los docentes, fue el ejercicio sistemático realizado en torno a la auto-inspección de sus propias prácticas (diagnóstico, recopilación de evidencias, trabajos personales y grupales) interpelando con ello las atribuciones que realizaban de su quehacer profesional. Así mismo, les permitió constatar que el docente puede incidir en la mejora del proceso enseñanza-aprendizaje de las Ciencias, sin desconocer que cada actor educativo tiene ámbitos de incidencia diferenciales en cada una las situaciones problemáticas abordadas. El proceso de investigación implicó poner en juego la voluntad de los docentes (el querer mejorar) y sus destrezas metacognitivas (el cómo), indagando y problematizando permanentemente su ejercicio profesional.

\subsubsection{Conformación de una comunidad de aprendizaje colaborativo}

$\mathrm{Al}$ inicio del proceso los docentes plantearon una serie de limitaciones para iniciar un proceso de formación continua, señalando, como muy importante, la falta de tiempo, la desacreditación del rol del profesor a nivel de la sociedad, el bajo nivel de autoeficacia respecto a su ejercicio laboral y procesos de formación continua deficientes. Al término de este primera etapa, los profesores han señalado como uno de los principales aportes de este proceso de formación el haber recuperado la confianza en su ejercicio profesional. Entre las potencialidades con las que cuenta este proceso, el trabajo colaborativo es la condición facilitadora mencionada con mayor frecuencia por los participantes, destacándose la heterogeneidad disciplinar del grupo, la participación de docentes provenientes de establecimientos de distintas dependencias (municipales, subvencionados, particulares), la diversidad de edad y de género. Esta condición posibilita contemplar una multiplicidad de perspectivas al abordar el fenómeno educativo, lo que enriqueció el aprendizaje y permitió derribar mitos que se presentaban en un inicio. También se destacan como condiciones que favorecen este Plan de Formación un clima de trabajo con apertura y flexibilidad al momento de compartir opiniones, discusiones de buen nivel temático entre profesionales, trabajo sistemático en torno a lecturas, reflexiones, trabajos individuales y grupales, entre otras.

Un aspecto fundamental señalado es la posibilidad de transferir los aprendizajes del proceso de formación continua a los contextos educativos en los cuales se desempeñan, para lo que es necesario realizar un aprendizaje explícito (Pozo, 2003), en la línea de dotar las representaciones que poseemos de un nuevo significado. 
"Tú te das cuenta que estos procesos intuitivos tienen un fundamento, hay alguien que ha escrito de esto y evidencia que esto viene a reafirmar aquello que uno puede estar haciendo mejor o peor, pero intuitivamente, y creo que la clave está en esta reflexión profesional" (FG1 427-433). ${ }^{5}$

La oportunidad de compartir experiencias entre pares promueve la transferencia al ampliar los repertorios de acción pedagógica y fortalecer las habilidades metacognitivas:

"Fundamentalmente el ser capaz de observar otras visiones sobre lo mismo que yo pienso, me refiero a que yo he aprendido con los otros sobre lo que hemos estado hablando, por ejemplo, sobre el concepto de Ciencia. Ha habido un aprendizaje de mi parte sobre mis prácticas que puedo llevar al plano de los alumnos, pero en el fondo me doy cuenta que son el mismo plano, que lo que yo trato de hacer como profesor es que ellos traten de hacer lo mismo que yo hago aquí con mis colegas..." (FG1 171-178).

Se señala también el lograr resituar el valor del aprendizaje de las Ciencias, destacando el formar ciudadanos críticos informados y que relativicen el saber.

Al comienzo de este trabajo se planteó la necesidad de generar en el docente una postura indagatoria y reflexiva respecto de sus prácticas como una manera de transitar hacia la transformación de sus prácticas de enseñanza y así responder a las actuales demandas de la educación científica.

Previo a poder desarrollar una postura indagatoria en el docente, y en consideración a la gran distancia que hay entre el mundo académico y la realidad del aula, se vuelve necesario construir dominios compartidos conceptuales y experienciales que sirvan de "piso común" para la problematización de las propias prácticas. Junto con ello, aparecen tres elementos que facilitarían la adquisición de una postura indagatoria frente a las prácticas: (a) el potenciamiento del razonamiento científico del docente, (b) la generación

Figura 1. Variables de proceso para la transformación de las prácticas

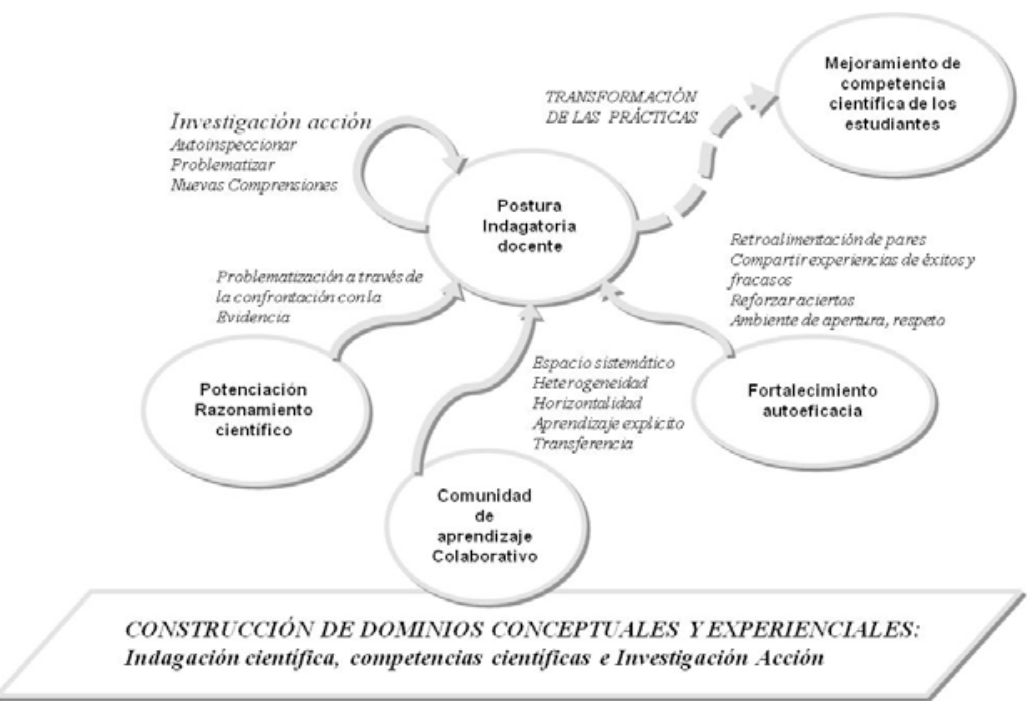

$5 \quad$ FG1: Focus group. 
de una comunidad de aprendizaje colaborativo y (c) el fortalecimiento de la autoeficacia docente. La Figura 1 resume estos resultados.

\section{DISCUSIÓN}

Es complejo revisar en detalle el proceso de ocho meses de trabajo, sin embargo, el equipo de Investigación-Acción posee la convicción de que el trabajo colaborativo, en alianza Universidad-Escuela, es la forma de generar transformaciones y de abordar el proceso de formación continua docente. El ejercicio reflexivo colaborativo, deliberativo, bien documentado, de su quehacer y del estado actual de la enseñanza de las Ciencias, imprime intencionalidad educativa al quehacer del profesor amplificando su capacidad de transformar sus prácticas. Dado lo anterior, se coincide con Warford (2011), quien refiere que el conocimiento del profesor es inherentemente situado y mediado, dentro de una interacción emergente y dinámica, donde la promoción de diálogos internos y el confluir de experiencias de enseñanza previa, elementos teóricos y las prácticas locales, se vuelve relevante. En este sentido, se requiere elaborar un plan de formación continua que no separe la teoría (los conceptos científicos y las actualizaciones en el campo de la didáctica de las Ciencias) de las prácticas pedagógicas (conceptos experienciales) con que cuenta el profesor.

Por otra parte, se debe considerar que los profesores traen a las instancias de formación continua un conjunto de recursos y un esquema cultural propio de su educación profesional, el que puede diferir del de los formadores de formadores (Milne et al., 2006), por lo que, en primer lugar, la instancia de formación continua debe convertirse en un sitio de negociación y construcción de nuevos significados comunes, lo que puede demandar tiempo y sistematicidad.

Finalmente, estos hallazgos coinciden con algunos de los aspectos que Loucks-Horsley et al. (1996) y Park-Rogers et al. (2007) plantean respecto de los procesos de desarrollo profesional efectivo en docentes de Ciencia: el orientarse por una imagen de enseñanza efectiva de las Ciencias, la cual incluye, entre otros, un énfasis en el aprendizaje a través de la Indagación Científica, la generación de comunidades de aprendizaje y de redes de colaboración, el desarrollo de conocimientos y habilidades en los docentes y la modelización de los métodos instruccionales. Esto implica generar oportunidades para que los docentes expliciten sus ideas previas y las cuestionen, generando un alto nivel de disonancia cognitiva que lleve a la construcción de nuevos significados a partir de un proceso colectivo. Todo lo anterior, debiera generar las condiciones necesarias para la transformación de las propias prácticas, con miras a mejorar los niveles de Competencia Científica en el alumnado.

Se espera que este trabajo pueda servir de insumo para los procesos de diseño de programas de formación continua, en particular en el ámbito de la formación de profesores de Ciencia secundarios.

\section{REFERENCIAS BIBLIOGRÁFICAS}

Abell, S., Smith, D. \&. Volkmann, M. (2006). Inquiry in Science Teacher Education. En Flick, L y N. Lederman (Eds.), Scientific inquiry and the nature of science: Implications for teaching, learning, and teacher education (pp. 389-425). Netherlands: Springer. 

PROFESORES SECUNDARIOS DE CIENCIAS...

Albertini, R., Cárdenas-Jirón, G., Babel, J., Díaz, G., Eyzaguirre, J., Labra, A. y Lewin, R. (2005). Enseñanza de las Ciencias a nivel escolar y formación en Ciencia en el pregrado universitario. En Ureta, T., Babul, J., Martínez, S., y Allende, J., Análisis y Proyecciones de la Ciencia Chilena 2005. Web: http://www.academia-Ciencias.cl/?module=investig.

Bandura, A. (1987). Pensamiento y acción. Fundamentos sociales. Barcelona: Martínez Roca.

Cochran-Smith, M. y Lytle, S. (2003). Más allá de la certidumbre: adoptar una actitud indagadora sobre la práctica. En Lieberman, A. y Millar, L. (Eds.), La indagación como base de la formación del profesorado y la mejora de la educación. Barcelona: Ediciones Octaedro.

Coll, C., Palacios, J. y Marchesi, A. (2004). Desarrollo psicológico y educación, 2. Psicología de la educación escolar. Madrid: Alianza Editorial.

Copello, M. y Sanmarti, N. (2001). Fundamentos de un modelo de formación permanente del profesorado de Ciencias centrado en la reflexión dialógica sobre las concepciones y las prácticas. Enseñanza de las Ciencias, vol. 19, n. 2, 269-283.

Elliot, J. (2010). La Investigación-Acción en Educación. Madrid: Morata.

Furio, C. y Carnicer, J. (2002). El desarrollo profesional del profesor de Ciencias mediante tutorías de Grupos Cooperativos. Estudio de ocho casos. Enseñanza de las Ciencias: revista de investigación y experiencias didácticas, vol. 20, n. 1, 47-74.

Garduño, L., Carrasco, M. y Raccanello, K. (2010). Los formadores de docentes y la autoeficacia para la enseñanza en una muestra de escuelas normales en el estado de puebla. Perfiles Educativos, vol. 32, n. 127, 85-104.

González-Weil, C., Cortéz, M., Bravo, P., Ibaceta, Y., Cuevas, K., Quiñones, P., Maturana, J. y Abarca, A. (2012) La indagación científica como enfoque pedagógico: estudio sobre las prácticas innovadoras de docentes de ciencia en EM (Región de Valparaíso). Estudios Pedagógicos, vol. 38, n. 2, 85-102.

Holbrook, J. \& Rannikmae, M. (2009). The meaning of scientific literacy. International Journal of Environmental \& Science Education, vol. 4, n. 3, 275-288.

Labra, P., Montenegro, G., Iturra, C. y Fuentealba, R. (2005). La investigacion-acción como herramienta para lograr coherencia de acción en el proceso de práctica profesional durante la formación inicial docente. Estudios Pedagógicos, vol. 31, n. 2, 137-143.

Lavonen, J. \& Laaksonen, S. (2009). Context of teaching and learning school science in Finland: Reflections on PISA 2006 Results. Journal of Research in Science Teaching, vol. 46, n. 8, 922-944.

Loucks-Horsley, S., Styles, K., \& Hewson, P. (1996). Principles of effective professional development for mathematics and science education: A synthesis of standards. National Institute for Science Education, vol. 1, n. 1, 1-6.

Milne, C., Scantlebury, K. \& Otieno, T. (2006). Using sociocultural theory to understand the relationship between teacher change and a science-based profesional education program. Cultural Studies of Science Education, n. 1, 325-352.

Moliner, L. y Loren, C. (2010). La formación continua como proceso clave en la profesionalización docente: Buenas prácticas en Chile. Revista Latinoamericana de Inclusión Educativa, vol. $4, n .1,25-44$.

Montecinos, C. (2003). Desarrollo profesional docente y aprendizaje colectivo. Revista Psicoperspectivas, vol. 2, n. 1, 105-128.

Montecinos, C., Soliz, M. y Gabriele, A. (2001). Aprendiendo a enseñar a través de la investigaciónacción. Paideia Revista de Educación, vol. 30/31, 37-50.

OECD (2006). Assessing Scientific, Reading and Mathematical Literacy: A framework for PISA 2006. Web: http://www.oecd.org/dataoecd/63/35/37464175.pdf

Park Rogers, M., Abell, S., Lannin, J., Wang, Ch., Musikul, K., Barker, D., Dingman, S. (2007). Effective professional development in science and mathematics education: teachers' and facilitators' views. International Journal of Science and Mathematics Education, vol. 5, 507-532. 
Pasmanik, D. y Cerón, R. (2005). Las prácticas pedagógicas en el aula como punto de partida para el análisis del proceso enseñanza-aprendizaje: un estudio de caso en la asignatura de química. Estudios Pedagógicos, vol. 31, n. 2, 71-87.

Pozo, J. (1996). Aprendices y maestros: la nueva cultura del aprendizaje. Madrid: Alianza Editorial. Pozo, J. (2003). Adquisición de conocimiento. Madrid: Ediciones Morata.

Tytler, R. (2007). Re-imagining Science Education. Engaging students in science for Australia's future. Victoria: Australian Council for Educational Research.

Vezub, L. (2007). La formación y el desarrollo profesional docente frente a los nuevos desafíos de la escolaridad. Profesorado. Revista de Currículum y Formación de Profesorado, vol. 11, n. 1. Web: http://www.ugr.es/ recfpro/rev111ART2.pdf

Warford, M. (2011). The zone of proximal teacher development. Teaching and Teacher Education, n. $27,252-258$. 